\title{
Distribution of lymphocele following lymphadenectomy in patients with gynecological malignancies
}

Soo Youn Song, MD, PhD, Mia Park, MD, Byung Hun Kang, MD, PhD, Jung Bo Yang, MD, PhD, Young Bok Ko, MD, PhD, Mina Lee, MD, PhD, Ki Hwan Lee, MD, PhD, Heon Jong Yoo, MD, PhD

Department of Obstetrics and Gynecology, Chungnam National University Hospital, Chungnam National University School of Medicine, Daejeon, Korea

\section{Objective}

This study identified the distribution of lymphocele, as well as the factors associated with lymphocele formation, in patients undergoing pelvic and/or para-aortic lymph node dissection (PLND and/or PALND) for gynecologic malignancies.

\section{Methods}

This study was retrospective, and data were collected from patients who underwent surgical procedures including lymphadenectomy due to gynecologic malignancies from March 2013 to May 2016. Lymphocele was defined by postoperative computer tomography within 2 weeks after surgery.

Results

A total of 116 patients underwent lymphadenectomy, of whom, 47 (42.0\%) developed lymphocele and 14 (12.1\%) had symptomatic lymphocele formation. The affecting factors of lymphocele formation were PLND concomitant with PALND and a large amount of blood loss $\geq 600 \mathrm{~mL}(P=0.030$ and $P=0.006$, respectively). All clinical factors were not significantly different between patients with symptomatic and asymptomatic lymphocele. Lymphocele developed more frequently in the left side $(67.1 \%)$ of the body compared to the right side $(48.7 \%)$, and in the pelvic area $(75.9 \%)$ compared to the para-aortic area $(24.1 \%, P<0.001$, both).

\section{Conclusion}

Lymphocele formation is more prevalent in the left and pelvic area of the body compared to the right and paraaortic side. PLND concurrent with PALND and large amounts of blood loss were significant risk factors for lymphocele formation.

Keywords: Lymphocele; Lymph node excision; Genital neoplasms, female

\section{Introduction}

Lymphadenectomy is the removal of one or more groups of lymph nodes by surgery, and is a crucial part of diagnosis and treatment of gynecological malignancies such as cervical, endometrial or ovarian cancer [1]. However, lymphadenectomy can induce complications such as lymphocele, lymphedema and bleeding [2]. Lymphocele formation is the most common complication following injury to lymphatic vessels following lymphadenectomy [3]. Although most lymphocele are asymptomatic and are usually detected incidentally, symptomatic lymphocele occur in about $5-34.5 \%$ of cases [4]. Symptomatic lymphocele can seriously affect patients' quality
Received: 2020.04.22. Revised: 2020.07.08. Accepted: 2020.07.20. Corresponding author: Heon Jong Yoo, $\mathrm{MD}, \mathrm{PhD}$

Department of Obstetrics and Gynecology, Chungnam National University Hospital, Chungnam National University School of Medicine, 33 Munhwa-ro, Jung-gu, Daejeon 34998, Korea E-mail: bell4184@gmail.com https://orcid.org/0000-0003-4808-3450

Articles published in Obstet Gynecol Sci are open-access, distributed under the terms of the Creative Commons Attribution Non-Commercial License (http://creativecommons. org/licenses/by-nc/3.0/) which permits unrestricted non-commercial use, distribution, and reproduction in any medium, provided the original work is properly cited.

Copyright $\odot 2020$ Korean Society of Obstetrics and Gynecology 


\section{Obstetrics \& Gynecology Science}

Soo Youn Song, et al. Distribution of lymphocele

of life and interfere with adjuvant treatment [4].

A variety of clinical and surgical factors might be correlated with the occurrence of lymphocele: body mass index (BMI); cancer type; postoperative radiation therapy; the number of lymph nodes obtained; the number of tumor-positive lymph nodes; surgical methods; radical surgery; and the degree of lymphadenectomy [5]. However, the correlation between these factors and lymphocele formation showed conflicting results $[4,6-8]$. Until now, most studies have focused on the causes underlying lymphocele. There is a paucity of data discussing the distribution of lymphocele after pelvic and/or para-aortic lymph node dissection (PLND and/or PALND) in gynecological malignancies. The purpose of this study was to identify the distribution of lymphocele and factors associated with lymphocele formation in patients undergoing PLND and/or PALND in gynecological cancer.

\section{Materials and methods}

\section{Patients}

Data were collected from patients who underwent surgical procedures including PLND and/or PALND due to cervical, ovarian, or endometrial cancers in Chungnam National University Hospital from March 2013 to May 2016. Patients with gynecological malignancy who failed to undergo lymphadenectomy and those with inoperable medical conditions and failure to undergo postoperative imaging evaluation were excluded from this study. Patients' data such as basic demographics, cancer stage and histology, and detailed surgical information including the extent of surgery, type of lymphadenectomy, number of removed and positive lymph nodes, neoadjuvant or adjuvant treatments, duration of hospital stay, size of lymphocele and complications associated with lymphoceles were collected by reviewing the medical records. All the patients' records of postoperative symptoms were collected, and the lymphocele was defined as symptomatic if the symptoms were possibly related to the lymphocele such as flank pain due to hydronephrosis, urinary urgency, venous thrombosis and lymphedema, infection, or pain of the lower extremities.

\section{Pre and postoperative work-up}

Preoperative work-up to evaluate the extent of the disease included imaging studies (computed tomography [CT], mag- netic resonance imaging, and positron emission tomographyCT), endoscopic work-up (esophagogastroduodenoscopy, colonoscopy, proctoscopy, and cystoscopy), and tumor markers (carcinoembryonic antigen, squamous cell carcinoma, cancer antigen [CA] 125, and CA 19-9), as needed within 1 month. Postoperative CT follow-up was carried out in all patients within 2 weeks after surgery. All patients were followed with a CT scan every 6 months for 2 years. A lymphocele was defined as a low-attenuated fluid-filled cyst without an epithelial lining. The largest diameter and the location of lymphoceles were recorded. All of the patients were followed up for at least 6 months after surgery.

\section{Surgical procedures}

All patients underwent primary surgery to remove tumors either primarily or after neoadjuvant treatment. PLND and/ or PALND were performed as part of staging and treatment in patients with endometrial, cervical and ovarian cancers using bipolar energy by one of 2 experienced gynecological oncologists. Lymphadenectomy was defined as the excision of all fibro-fatty tissues along the surrounding blood vessel. The procedure was carried out using either laparotomy or laparoscopy; however, the extent and the performance

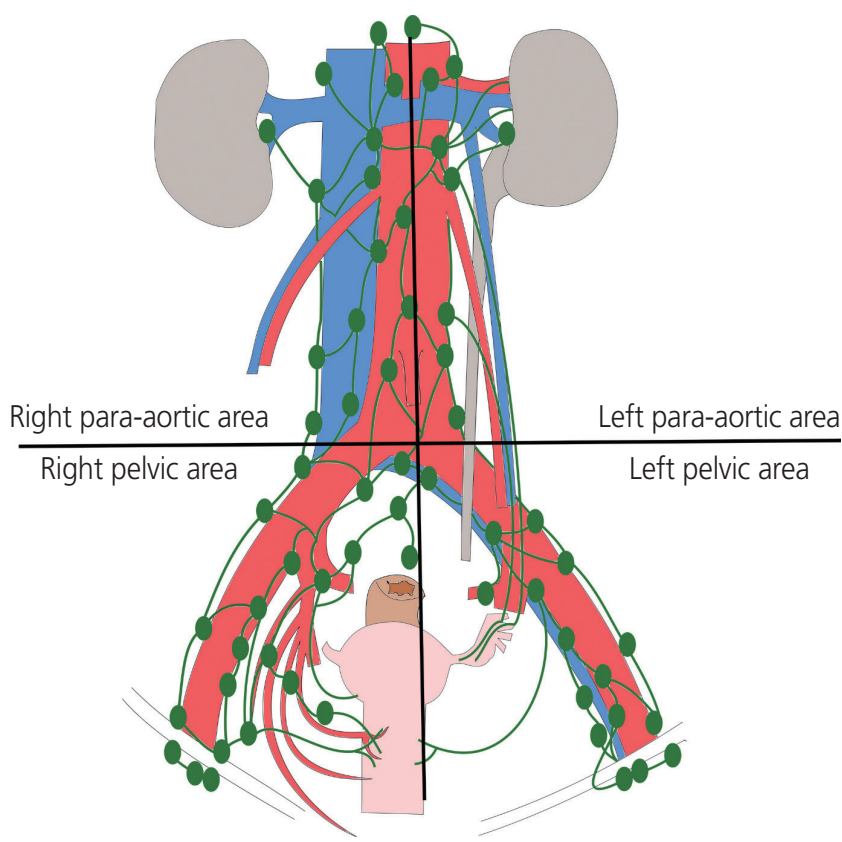

Fig. 1. Area of lymphadenectomy. Lymphadenectomy was divided into 4 sections based on aortic bifurcation and aorta center; left pelvic area, right pelvic area, left para-aortic area, right para-aortic area. 


\section{Obstetrics \& Gynecology Science}

Vol. 63, No. 6, 2020

were identical in both approaches. In PLND, lymphatic tissue was obtained from the external iliac, common iliac, obturator and internal iliac regions from the vena circumflexa ilium profunda up to aortic bifurcation. In PALND, lymphatic tissue was obtained from the paracaval, interaortocaval, and paraaortic regions from the aortic bifurcation up to the level of both renal veins. In this study, the area in which lymphadenectomy was performed was divided into 4 sections based on aortic bifurcation and the center of aorta; the left pelvic area, right pelvic area, left para-aortic area, right para-aortic area (Fig. 1). A Jackson-Pratt drain was inserted in all patients and removed when the amount of fluid drained was less

Table 1. Comparison of patients with lymphocele and patients without lymphocele

\begin{tabular}{|c|c|c|c|}
\hline Characteristics & $\begin{array}{l}\text { Patients with lymphocele } \\
\qquad(n=47)\end{array}$ & $\begin{array}{l}\text { Patients without lymphocele } \\
\qquad(n=69)\end{array}$ & $P$-value \\
\hline Age & $52.9 \pm 11.4(31-76)$ & $52.9 \pm 11.4(13-75)$ & 0.713 \\
\hline BMI & $23.7 \pm 3.5(18-32)$ & $24.9 \pm 4.0(18-41)$ & 0.103 \\
\hline DM & $4(8.5)$ & $5(7.2)$ & 0.533 \\
\hline Hypertension & $9(19.1)$ & $16(23.2)$ & 0.389 \\
\hline Prior abdominal surgery & $13(27.7)$ & $24(34.8)$ & 0.274 \\
\hline Type of cancer & & & 0.045 \\
\hline Cervical cancer & $8(17.0)$ & $25(36.2)$ & \\
\hline Ovarian cancer & $24(51.1)$ & $22(31.9)$ & \\
\hline Endometrial cancer & $15(31.9)$ & $22(31.9)$ & \\
\hline Stage & & & 0.516 \\
\hline I & $29(63.0)$ & $48(67.2)$ & \\
\hline$\|$ & $3(6.5)$ & $5(7.5)$ & \\
\hline III & $10(21.7)$ & $8(11.9)$ & \\
\hline IV & $4(8.7)$ & $9(13.4)$ & \\
\hline Neoadjuvant CTx & $5(10.6)$ & $8(11.6)$ & 0.697 \\
\hline Postoperative CTx & $28(59.6)$ & $34(49.3)$ & 0.424 \\
\hline Postoperative RTx & $10(21.3)$ & $19(27.5)$ & 0.511 \\
\hline Approach method & & & 0.016 \\
\hline Laparoscopy & $9(19.1)$ & $28(40.6)$ & \\
\hline Laparotomy & $38(80.9)$ & $41(59.4)$ & \\
\hline \multicolumn{4}{|l|}{ Operation type } \\
\hline PLND & $46(97.9)$ & $68(98.6)$ & 0.648 \\
\hline PLND with PALND & $40(85.1)$ & $33(47.8)$ & $<0.001$ \\
\hline Surgical glue apply & $47(100.0)$ & $67(97.1)$ & 0.352 \\
\hline Operation time (min) & $329.64 \pm 129.40(132-635)$ & $306.61 \pm 142.30(91-702)$ & 0.377 \\
\hline Estimated blood loss (mL) & $814.9 \pm 1,100.7(100-6,000)$ & $458.8 \pm 543.2(20-3,000)$ & 0.044 \\
\hline No. of removed lymph nodes & $37.3 \pm 19.8(3-92)$ & $29.5 \pm 16.0(7-73)$ & 0.020 \\
\hline No. of positive lymph nodes & $1.9 \pm 4.4(0-23)$ & $0.9 \pm 2.1(0-12)$ & 0.161 \\
\hline Lymphorrhea (mL) & $6,774 \pm 6,527(402-26,995)$ & $5,272 \pm 9,557(0-60,926)$ & 0.350 \\
\hline Duration of hospital stay (day) & $20.7 \pm 8.4(9-44)$ & $15.8 \pm 8.2(3-44)$ & 0.044 \\
\hline
\end{tabular}

Data are presented as mean \pm standard deviation (range) or number (\%).

BMI, body mass index; DM, diabetes mellitus; CTx, chemotherapy; RTX, radiotherapy; PLND, pelvic lymph node dissection; PALND, para-aortic lymph node dissection. 


\section{Obstetrics \& Gynecology Science}

Soo Youn Song, et al. Distribution of lymphocele

than $100 \mathrm{~mL}$ per day for 2 consecutive days. A few patients with a large amount of lymphorrhea were discharged from the hospital without removing the Jackson-Pratt drain and were asked to maintain records of the amount of drainage at home. Adjuvant therapies such as chemotherapy or radiotherapy were performed in patients depending on malignancies 3 to 4 weeks postoperatively.

\section{Statistical analysis}

The characteristics of patients with and without lymphocele were compared using $t$-test for continuous variables and $\chi^{2}$ test (or Fisher's exact test when the expected frequency within any cell was less than 5) for categorical variables. The incidence of lymphocele formation in each section after lymphadenectomy, and the number of harvested lymph nodes was compared among different sites using 2 sample $t$-test and chi-squared test. A $P$-value of $<0.05$ was considered statistically significant. All analyses were performed using SPSS version 22.0 (IBM Corp., Armonk, NY, USA).

Table 2. Univariate and multivariate analysis of lymphocele formation

\begin{tabular}{|c|c|c|c|c|}
\hline \multirow{2}{*}{ Predictors } & \multicolumn{2}{|c|}{ Univariate } & \multicolumn{2}{|c|}{ Multivariate } \\
\hline & OR $(95 \% \mathrm{Cl})$ & $P$-value & OR $(95 \% \mathrm{Cl})$ & $P$-value \\
\hline BMl & & 0.848 & & \\
\hline$<25$ & 1.000 & & & \\
\hline$\geq 25$ & $1.059(0.671-1.670)$ & & & \\
\hline Age & & 0.611 & & \\
\hline$<55$ & 1.000 & & & \\
\hline$\geq 55$ & $1.214(0.576-2.560)$ & & & \\
\hline DM & & 0.533 & & \\
\hline No & 1.000 & & & \\
\hline Yes & $1.191(0.302-4.688)$ & & & \\
\hline Surgical hemostatic glue application & & 0.352 & & \\
\hline No & 1.000 & & & \\
\hline Yes & $0.971(0.932-1.011)$ & & & \\
\hline Surgical approach & & 0.016 & & 0.871 \\
\hline Laparoscopy & 1.000 & & 1.000 & \\
\hline Laparotomy & $1.458(1.102-1.929)$ & & $1.091(0.381-3.120)$ & \\
\hline Lymphadenectomy & & $<0.001$ & & 0.016 \\
\hline PLND only & 1.000 & & 1.000 & \\
\hline PLND with PALND & $5.637(2.301-13.807)$ & & $3.575(1.267-10.088)$ & \\
\hline No. of removed lymph nodes & & 0.067 & & \\
\hline$<40$ & 1.000 & & & \\
\hline$\geq 40$ & $1.433(0.976-2.103)$ & & & \\
\hline Estimated blood loss (mL) & & $<0.001$ & & 0.011 \\
\hline$<600$ & 1.000 & & 1.000 & \\
\hline$\geq 600$ & $5.867(2.373-14.503)$ & & 3.598 (1.349-9.599) & \\
\hline Type of cancer & & 0.346 & & \\
\hline Other types of cancer & 1.000 & & & \\
\hline Ovarian cancer & $1.685(0.569-4.987)$ & & & \\
\hline
\end{tabular}

OR, odds ratio; Cl, Confidence interval; BMI, body mass index; DM, diabetes mellitus; PLND, pelvic lymph node dissection; PALND, para-aortic lymph node dissection. 


\section{Obstetrics \& Gynecology Science}

Vol. 63, No. 6, 2020

\section{Results}

\section{Demographics}

A total of 116 patients underwent lymphadenectomy from March 2013 to May 2016. Basic characteristics of patients are listed in Table 1. Among 116 patients, 33 were diag- nosed with cervical cancer, 46 with ovarian cancer, and 37 with endometrial cancer. PLND was performed in all except 2 patients (1 patient with ovarian cancer, the other with endometrial cancer) who only underwent PALND. The number of patients who underwent PALND was 73. According to cancer subtype, $33.3 \%$ (11/33) of patients with cervical can-

Table 3. Comparison of patients with symptomatic and asymptomatic lymphocele

\begin{tabular}{|c|c|c|c|}
\hline Characteristics & $\begin{array}{l}\text { Symptomatic lymphocele } \\
\qquad(n=14)\end{array}$ & $\begin{array}{l}\text { Asymptomatic lymphocele } \\
\qquad(n=33)\end{array}$ & $P$-value \\
\hline Age & $52.1 \pm 10.3(35-70)$ & $54.4 \pm 11.9(31-76)$ & 0.386 \\
\hline BMI & $24.4 \pm 3.4(19-32)$ & $23.4 \pm 3.5(18-32)$ & 0.444 \\
\hline DM & $1(7.1)$ & $3(9.1)$ & 0.646 \\
\hline Hypertension & $2(14.3)$ & $7(21.2)$ & 0.437 \\
\hline Prior abdominal surgery & $2(14.3)$ & $11(33.3)$ & 0.150 \\
\hline Type of cancer & & & 0.956 \\
\hline Cervical cancer & $2(14.3)$ & $6(18.2)$ & \\
\hline Ovarian cancer & $7(50.0)$ & $17(51.5)$ & \\
\hline Endometrial cancer & $5(35.7)$ & $10(30.3)$ & \\
\hline Stage & & & 0.677 \\
\hline 1 & $10(71.4)$ & $19(59.4)$ & \\
\hline$\|$ & $1(7.1)$ & $2(6.3)$ & \\
\hline III & $3(21.4)$ & $7(21.9)$ & \\
\hline IV & $0(0)$ & $4(12.5)$ & \\
\hline Neoadjuvant CTx & $1(7.1)$ & $4(12.1)$ & 0.646 \\
\hline Postoperative CTx & $10(71.4)$ & $18(54.5)$ & 0.332 \\
\hline Postoperative RTx & $2(14.3)$ & $8(24.2)$ & 0.347 \\
\hline Approach & & & 0.263 \\
\hline Laparoscopy & $4(28.6)$ & $5(15.2)$ & \\
\hline Laparotomy & $10(71.4)$ & $28(84.8)$ & \\
\hline \multicolumn{4}{|l|}{ Operation type } \\
\hline PLND & $14(100)$ & $32(97.0)$ & 0.696 \\
\hline PALND & $11(78.6)$ & $29(87.9)$ & 0.358 \\
\hline Surgical glue apply & $14(100.0)$ & $33(100)$ & 0.352 \\
\hline Operation time & $308.0 \pm 108.2(192-504)$ & $338.8 \pm 137.9(132-635)$ & 0.508 \\
\hline Estimated blood loss (mL) & $621.4 \pm 553.6(100-2,000)$ & $897.0 \pm 1,262.5(100-6,000)$ & 0.425 \\
\hline No. of removed lymph nodes & $43.1 \pm 26.4(4-92)$ & $34.9 \pm 16.1(3-67)$ & 0.224 \\
\hline No. of positive lymph nodes & $1.5 \pm 3.7(0-14)$ & $2.0 \pm 4.7(0-23)$ & 0.864 \\
\hline Lymphorrhea (mL) & $6,320.5 \pm 4,594.6(1,327-19,152)$ & $6,966.3 \pm 7,248.5(402-26,995)$ & 0.709 \\
\hline Size of lymphocele (cm) & $8.3 \pm 3.0(4.5-13.2)$ & $7.9 \pm 3.9(2.2-17.3)$ & 0.735 \\
\hline Duration of hospital stay (day) & $21.7 \pm 8.3(10-37)$ & $20.3 \pm 8.5(9-44)$ & 0.397 \\
\hline
\end{tabular}

Data are presented as mean \pm standard deviation (range) or number (\%).

BMI, body mass index; DM, diabetes mellitus; CTx, chemotherapy; RTx, radiotherapy; PLND, pelvic lymph node dissection; PALND, para-aortic lymph node dissection. 


\section{Obstetrics \& Gynecology Science}

Soo Youn Song, et al. Distribution of lymphocele

cer, $95.7 \%$ (44/46) of patients with ovarian cancer, $48.6 \%$ $(18 / 37)$ of patients with endometrial cancer underwent PALND. The median number of lymph nodes removed by lymphadenectomy was 29 (range: 3-92). A total of 30 patients had lymph node metastasis.

A total of 47 patients (40.5\%) developed lymphocele identified by CT scan and the median time from surgery to diagnosis was 10.5 days (range: 6-242 days). There were no differences in BMI, age, diabetes mellitus, hypertension or history of previous abdominal surgery between patients with and without lymphocele. Stages of cancer according to each type of cancer were not significantly different between patients with and without lymphocele. There were no patients who received neoadjuvant radiotherapy, and percentages of patients who received neoadjuvant or adjuvant chemotherapy, and postoperative radiotherapy were not significantly different between the 2 groups. Laparotomy approach and PLND concurrent with PALND were associated with a significantly higher incidence of lymphocele formation than the laparoscopic approach and PLND alone $(P=0.016$ and $P<0.001$, respectively). No significant difference in the total amount of lymphorrhea was detected between the with lymphocele and without lymphocele groups $(6,774 \mathrm{~mL}$ in lymphocele group vs. 5,272 mL in no lymphocele group, $P=0.350$ ). A total of 14 patients were discharged from the hospital with Jackson-Pratt drain in situ. Patients with lymphocele formation had a significantly higher number of lymph nodes removed $(P=0.020)$, without any significant difference in the number of positive lymph nodes. Estimated blood loss during the operation as well as the duration of the hospital stay was significantly higher in patients with lymphocele ( $P=0.044$ and $P=0.044$, respectively, Table 1$)$.

\section{Risk factors for lymphocele formation}

BMI, age, diabetes mellitus, the use of surgical hemostatic glue, the number of removed lymph nodes, and the type of cancers did not affect lymphocele formation according to univariate analysis (Table 2). However, univariate analysis showed that factors such as a surgical approach with either laparoscopy or laparotomy $(P=0.016)$, the extent of lymphadenectomy such as PLND only or PLND with PALND $(P<0.001)$, and the estimated blood loss $<600 \mathrm{~mL}$ or $\geq 600 \mathrm{~mL}(P<0.001)$ were correlated with lymphocele formation. Multivariate analysis was performed with statistically significant variables in univariate analyses. The extent of lymphadenectomy and

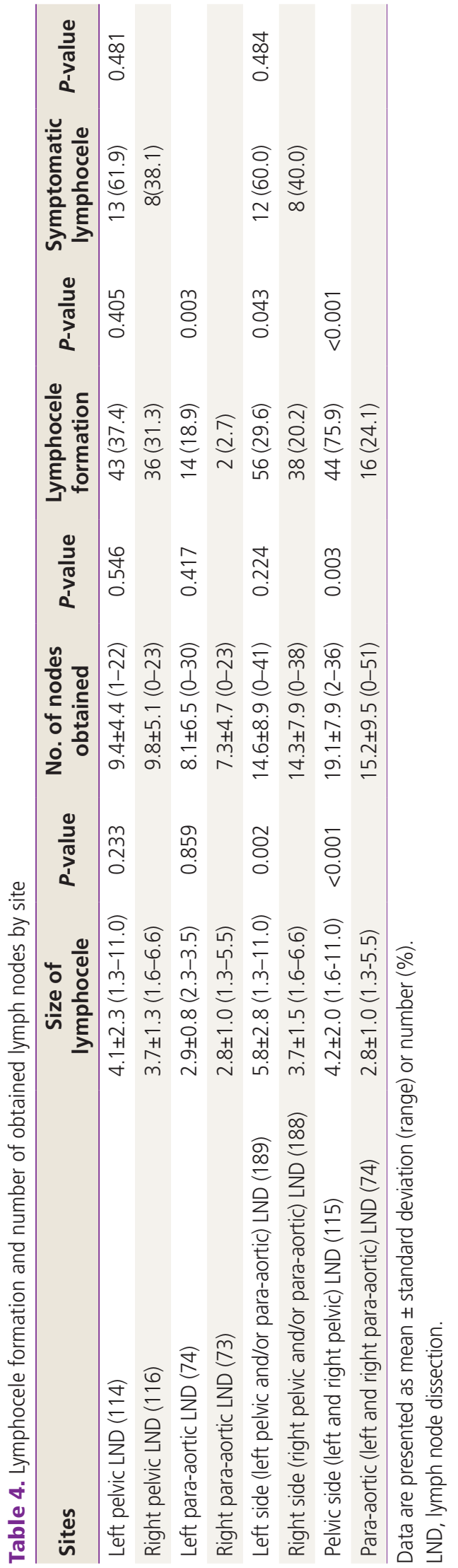




\section{Obstetrics \& Gynecology Science}

Vol. 63, No. 6, 2020

the large amount of blood loss were significantly associated with lymphocele formation in multivariate analysis $(P=0.016$ and $P=0.011$, respectively).

\section{Symptomatic and asymptomatic lymphocele}

Table 3 presents the comparison between patients with symptomatic and asymptomatic lymphocele. Among 47 patients with lymphocele, 14 patients (29.8\%) complained of symptoms related to lymphoceles and all symptomatic lymphocele were located in the pelvic area. The number of patients who received lymphocele drainage via percutaneous catheter insertion for symptom relief was 5 . The median time for lymphocele regression was 116 days (range: 6-618 days). Basic characteristics were comparable between the 2 groups, and all the factors including cancer stage and type, surgi- cal approach, extent of lymphadenectomy, operation time, estimated blood loss, and number of removed lymph nodes were not significantly different between patients with symptomatic and asymptomatic lymphocele. The incidence of symptomatic lymphocele formation was not significantly different between the left and right sides of the body (Table 4). There were no patients who experienced delay of scheduled adjuvant chemotherapy or radiotherapy due to symptomatic lymphocele formation.

\section{Lymphocele distribution}

Table 4 shows the comparative incidence of lymphocele formation after lymphadenectomy in the left and right sides, and the pelvic and para-aortic areas. The number of obtained lymph nodes were not different between the left and right

Table 5. Comparison of patients with left and right side lymphocele

\begin{tabular}{|c|c|c|c|}
\hline Characteristics & $\begin{array}{l}\text { Left side lymphocele } \\
\qquad(n=56)\end{array}$ & $\begin{array}{l}\text { Right side lymphocele } \\
\qquad(n=138)\end{array}$ & P-value \\
\hline Age & $53.04 \pm 10.90$ & $54.53 \pm 11.60$ & 0.528 \\
\hline $\mathrm{BMI}$ & $24.1 \pm 4.0$ & $24.1 \pm 3.5$ & 0.997 \\
\hline DM & $5(8.9)$ & $3(7.9)$ & 0.586 \\
\hline Hypertension & $8(14.3)$ & $7(18.4)$ & 0.775 \\
\hline Type of cancer & & & 0.677 \\
\hline Cervical cancer & $8(14.3)$ & $6(15.8)$ & \\
\hline Ovarian cancer & $33(58.9)$ & $19(50.0)$ & \\
\hline Endometrial cancer & $15(26.8)$ & $13(34.2)$ & \\
\hline Stage & & & 0.553 \\
\hline I & $31(57.4)$ & $26(70.3)$ & \\
\hline$\|$ & $7(13.0)$ & $2(5.4)$ & \\
\hline III & $12(22.2)$ & $7(18.9)$ & \\
\hline IV & $4(7.4)$ & $2(5.4)$ & \\
\hline Neoadjuvant CTx & $5(8.9)$ & $4(10.5)$ & 0.531 \\
\hline Postoperative CTx & $42(75.0)$ & $23(60.5)$ & 0.174 \\
\hline Postoperative RTx & $12(21.4)$ & $8(21.1)$ & 0.588 \\
\hline Approach method & & & 0.576 \\
\hline Laparoscopy & $10(17.9)$ & $7(18.4)$ & \\
\hline Laparotomy & $46(82.1)$ & $31(81.6)$ & \\
\hline Operation time (min) & $340.7 \pm 126.4$ & $324.7 \pm 122.6$ & 0.543 \\
\hline Estimated blood loss (mL) & $783.9 \pm 999.5$ & $746.1 \pm 897.2$ & 0.851 \\
\hline Lymphorrhea $(\mathrm{mL})$ & $6,465.6 \pm 5,865.2$ & $6,745.7 \pm 6,575.5$ & 0.829 \\
\hline Duration of hospital stay (day) & $20.5 \pm 7.1$ & $20.1 \pm 7.7$ & 0.739 \\
\hline
\end{tabular}

Data are presented as mean \pm standard deviation (range) or number (\%).

$\mathrm{BMI}$, body mass index; DM, diabetes mellitus; CTx, chemotherapy; RTx, radiotherapy. 


\section{Obstetrics \& Gynecology Science}

Soo Youn Song, et al. Distribution of lymphocele

side, but the size of the lymphocele was significantly larger in the left side compared to the right side $(5.8 \pm 2.8$ vs. $3.7 \pm 1.5$, $P=0.002$ ).

Pelvic lymphocele development was not significantly different between the left and right sides $(P=0.405)$, but paraaortic lymphocele formation was more prevalent in the left para-aortic area than in the right para-aortic area after PALND ( $P=0.003$ ). Left sided (left pelvic and/or para-aortic area, $29.6 \%$ ) lymphocele formation was dominant compared to the incidence of right-sided (right pelvic and/or para-aortic area, $20.2 \%$ ) lymphocele ( $P=0.043$ ). Lymphocele formation was significantly higher in the pelvic area $(75.9 \%)$ than in the para-aortic area $(24.1 \%)(P<0.001)$.

Other baseline characteristics were analyzed between left and right side lymphocele (Table 5). There were no significant difference between 2 groups in age, BMl, diabetes mellitus, hypertension, use of surgical hemostatic glue, number of removed lymph nodes, type of cancers, stage of cancer, approach method, amount of lymphorrhea or estimated blood loss.

\section{Discussion}

Distribution of lymphocele varies with pelvic and para-aortic lymphadenectomy in gynecological malignancies $[4,7,9]$. However, none of these studies emphasized the asymmetry of lymphocele distribution or compared the asymmetry. In this study, lymphocele developed more frequently in the left side of the body compared to the right side, and in the pelvic area compared to the para-aortic area. This kind of left-right asymmetry may be explained by differences in lymphatic drainage, and influence of peritoneal fluid movements, as Ayhan et al. [10] suggested. In their study, left side unilateral epithelial ovarian cancer was more likely to metastasize to the left iliac lymph nodes than the right side. In the present study, we found that lymphocele distribution was significantly different depending on the body area. However, further studies are needed to confirm these results.

Many studies showed that a variety of clinical factors affect lymphocele formation after PLND and/or PALND in gynecological malignancies $[2,4,6,8]$. In the present study, factors such as PLND concurrent with PALND and estimated blood loss $\geq 600 \mathrm{~mL}$ were significant risk factors for lymphocele formation. Correlation between performances of PALND with lymphocele formation was consistent with previous reports $[2,4,6,8]$. The estimated blood loss during the operation was only assessed by Tsuda et al. [11], who did not report a higher blood loss as a risk factor for lymphocele formation. This discrepancy might be attributed to the significant difference in hemostatic methods, such as tie ligation or use of an electrothermal bipolar vessel sealing device [11]. Further prospective studies without such confounding factors are needed to confirm the results.

Factors including BMI, the positivity of removed lymph nodes, and postoperative radiation therapy were not correlated with lymphocele formation in this study. The correlation between these factors and lymphocele formation has been disputed in many studies. Kim et al. [1] reported significantly higher BMI in patients with lymphoceles. However, Zikan et al. [4] reported the opposite result. The positivity of removed lymph nodes was correlated with lymphocele formation by Zikan et al. [4] but not by Tsuda et al. [11]. Postoperative radiotherapy was reported as a risk factor by Kim et al. [7] and Zikan et al. [4] but not by Achouri et al. [6] or Tsuda et al. [11]. The correlation between these factors and lymphocele formation showed conflicting results. These results should be further confirmed in prospective studies.

The current study reported that symptomatic lymphocele was not related to the size of the lymphocele and no asymmetry of distribution was found. Symptomatic lymphocele formation was not associated with factors such as cancer type, radical hysterectomy or total number of lymph nodes obtained, which represent 3 major risk factors for symptomatic lymphocele formation reported by Zikan et al. [4]. This discrepancy might be due to the smaller sample size of this study. Further prospective studies with larger populations are needed to confirm these results.

The study limitation relates to the small number of patients reviewed. Further, since this study was retrospective, a selection bias and other confounders are inevitable. However, to the best of our knowledge, this study was the first to analyze the asymmetrical distribution of lymphocele between left vs. right and pelvic vs. para-aortic regions of the body. Possible asymmetry of lymphocele distribution as well as the risk factors for lymphocele formation investigated in this study facilitate the identification of patients at risk of developing lymphocele and early detection of lymphocele formation. Moreover, this information could also be helpful in preventing lymphocele formation, by minimizing blood loss during 


\section{Obstetrics \& Gynecology Science}

Vol. 63, No. 6, 2020

surgery wherever possible. Lymphocele formation leads to longer hospital stays, as shown in this study. Therefore, early detection and appropriate management of lymphocele, such as by drainage, result in improved postoperative outcomes in patients.

In conclusion, lymphocele formation is more prevalent in the left and pelvic areas of the body compared to the right and para-aortic side, respectively. PLND concurrent with PALND and large amounts of blood loss were significant risk factors for lymphocele formation. Further prospective studies with larger populations are needed to confirm these results.

\section{Acknowledgments}

This work was supported by a research fund from Chungnam National University.

\section{Conflict of interest}

No potential conflict of interest relevant to this article was reported.

\section{Ethical approval}

This retrospective study was approved by the Institutional Review Board of Chungnam National University Hospital, Korea (2017-06-016).

\section{References}

1. Kim YH, Shin HJ, Ju W, Kim SC. Prevention of lymphocele by using gelatin-thrombin matrix as a tissue sealant after pelvic lymphadenectomy in patients with gynecologic cancers: a prospective randomized controlled study. J Gynecol Oncol 2017;28:e37.
2. Tinelli A, Mynbaev OA, Tsin DA, Giorda G, Malvasi A, Guido $M$, et al. Lymphocele prevention after pelvic laparoscopic lymphadenectomy by a collagen patch coated with human coagulation factors: a matched case-control study. Int J Gynecol Cancer 2013;23:956-63.

3. Mori N. Clinical and experimental studies on the socalled lymphocyst which develops after radical hysterectomy in cancer of the uterine cervix. J Jpn Obstet Gynecol Soc 1955;2:178-203.

4. Zikan M, Fischerova D, Pinkavova I, Slama J, Weinberger $V$, Dusek $L$, et al. A prospective study examining the incidence of asymptomatic and symptomatic lymphoceles following lymphadenectomy in patients with gynecological cancer. Gynecol Oncol 2015;137:291-8.

5. Weinberger V, Cibula D, Zikan M. Lymphocele: prevalence and management in gynecological malignancies. Expert Rev Anticancer Ther 2014;14:307-17.

6. Achouri A, Huchon C, Bats AS, Bensaid C, Nos C, Lécuru F. Complications of lymphadenectomy for gynecologic cancer. Eur J Surg Oncol 2013;39:81-6.

7. Kim HY, Kim JW, Kim SH, Kim YT, Kim JH. An analysis of the risk factors and management of lymphocele after pelvic lymphadenectomy in patients with gynecologic malignancies. Cancer Res Treat 2004;36:377-83.

8. Petru E, Tamussino K, Lahousen M, Winter R, Pickel H, Haas J. Pelvic and paraaortic lymphocysts after radical surgery because of cervical and ovarian cancer. Am J Obstet Gynecol 1989;161:937-41.

9. Scholz HS, Petru E, Benedicic C, Haas J, Tamussino K, Winter R. Fibrin application for preventing lymphocysts after retroperitoneal lymphadenectomy in patients with gynecologic malignancies. Gynecol Oncol 2002;84:43-6.

10. Ayhan A, Yüksel H, Dursun P. The spread pattern of right and left epithelial ovarian cancers. Eur J Gynaecol Oncol 2010;31:654-7.

11. Tsuda N, Ushijima K, Kawano K, Takemoto S, Nishio S, Sonoda $G$, et al. Prevention of lymphocele development in gynecologic cancers by the electrothermal bipolar vessel sealing device. J Gynecol Oncol 2014;25:229-35. 